\title{
IMPLANTAÇÃO DE BIM NA GRADUAÇÃO EM ENGENHARIA CIVIL DA UNIVERSIDADE FEDERAL DE PELOTAS: UMA ABORDAGEM PEDAGÓGICA
}

\author{
Victor Hugo Santos de Oliveira - victorwellcha@gmail.com \\ Universidade Federal de Pelotas \\ Praça Domingos Rodrigues \\ 96010-450 - Pelotas - Rio Grande do Sul
}

Klaussen Prestes Paim - klaussenpaim@hotmail.com

Universidade Federal de Pelotas

Praça Domingos Rodrigues

96010-450 - Pelotas - Rio Grande do Sul

Klaus Machado Theisen - theisenkm@yahoo.com.br

Universidade Federal de Pelotas

Praça Domingos Rodrigues

96010-450 - Pelotas - Rio Grande do Sul

Resumo: A metodologia BIM vem se destacando pela sua praticidade e ganhos, tanto em valor quanto em tempo de planejamento, no desenvolvimento de projetos em construção civil. Sendo uma competência requerida por grandes empresas da área e pelo poder público, a implementação do ensino de tal metodologia durante a graduação se torna mais do que necessária para a formação de engenheiros civis qualificados e capazes de poderem atuar com as ferramentas tecnológicas mais adequadas a seu dispor, otimizando os processos intrínsecos a profissão. Com a proposta de inserir o BIM no currículo da graduação em engenharia civil da Universidade Federal de Pelotas, investigou-se entre a comunidade do curso suas considerações sobre a metodologia. Ao comparar com o corpo docente, os alunos já possuíam mais familiaridade com os conceitos de BIM, provavelmente por interagirem com maior intensidade com o mercado de trabalho. Toda a comunidade acadêmica do curso concordou que o ensino de BIM pode promover o desenvolvimento de competência relevante para a formação dos alunos, além da votação unânime entre os discentes pela opção de ter o BIM abordado em aula. A maioria dos professores se mostraram positivos a se qualificarem para ministrar aulas sobre a metodologia. A comunidade acadêmica preferiu que o BIM fosse abordado ao longo da graduação, em diversas matérias. Enfatiza-se a necessidade de capacitação e apoio do corpo docente para a implementação bem sucedida da metodologia, sendo estes os que possuem a capacidade de atualizar o currículo do curso e engajar o restante da comunidade.

Palavras-chave: BIM. Implementação. Currículo.

\section{INTRODUÇÃO}

A metodologia Building Information Modeling (BIM) está deixando de ser algo opcional e se tornando cada vez mais uma necessidade. Instituições de ensino e órgãos públicos estão aos poucos tornando o uso da plataforma algo obrigatório. No Brasil, o uso do BIM para projetos e construções se tornará obrigatória a partir de 2021, com estados como 
Santa Catarina sendo pioneiros nessa questão, onde desde 2019 todas as licitações de obras públicas são projetadas com o uso do BIM (INBEC, 2018).

Existe uma grande deficiência na estratégia para implementar essa metodologia, sendo motivo de grande debate em seus pontos cruciais, como a capacidade de quem ensina, além de como implementar o ensino do método no curso. Para a implementação de um currículo que lide com BIM é de extrema importância identificar as competências e capacidades de quem irá ministrar o ensino da plataforma e assim planejar de acordo com a ementa proposta. (BARISON \& SANTOS, 2010).

Nas universidades brasileiras observa-se dois modelos que estão sendo aplicados nos currículos dos cursos de Engenharia e Arquitetura: uma integrada com as disciplinas técnicas dos cursos e uma adoção pontual que foca em disciplinas específicas do curso, cada uma delas com suas vantagens e desvantagens as quais demandam adaptações variadas (BARISON \& SANTOS, 2010; BARISON \& SANTOS, 2011).

Outra dificuldade enfrentada na implementação de BIM é a interdisciplinaridade entre os cursos da área. $\mathrm{Na}$ maioria dos casos as universidades não têm essa prática entre departamentos, o que se agrava muito mais quando se observa a necessidade de integração dos docentes tornando isto em um dos maiores obstáculos do ensino de BIM. Segundo Checcucci et al.(2013), o melhor cenário possível é um onde a instituições consideram estas condições desde o início do processo de implementação, minimizando assim as dificuldades encontradas com a inserção da metodologia no curso.

A partir da revisão bibliográfica do assunto foi-se determinado os pontos que devem ser estudados para a realização do diagnóstico da implementação da metodologia BIM no curso superior de Engenharia Civil da Universidade Federal de Pelotas, com ênfase no aspecto pedagógico. Analisando estes pontos será possível produzir um parecer analítico sobre esta questão, fundamentado também nas necessidades dos componentes da instituição, determinadas pelo diagnóstico supracitado com auxílio de um questionário. Se valendo dessas informações, a determinação da aplicabilidade do BIM no curso se baseará pelos dados inferidos nos questionários e nas conclusões da bibliografia da área.

\section{REVISÃO BIBLIOGRÁFICA}

É importante quando se discute BIM ter em mente o conceito da metodologia, para que não haja confusões em sua abordagem. Building Information Modeling (BIM) é um conceito que envolve a modelação das informações do edifício, criando um modelo digital integrado de todas as especialidades, e que abrange todo o ciclo de vida da edificação, desde o estudo de viabilidade, a projetação, o planejamento da construção, a construção, a operação, a manutenção, até a demolição ou a requalificação, com a modelação 3D paramétrica e a interoperabilidade como características essenciais que suportam esse conceito (CHECCUCCI et al., 2013).

Como citado acima, a interoperabilidade exerce um papel extremamente importante quando se trabalha com BIM para que se possa trabalhar com informações proveniente de várias fontes, sendo assim a possibilidade de compartilhar abertamente e facilmente as informações em formatos genéricos, sem as restrições do software se torna algo crítico. Segundo Azevedo (2009), a troca de informações entre esses programas deve ser possível sem nenhum tipo de degradação dos dados, para assim evitar o retrabalho de inserção de elementos perdidos ou corrompidos, garantindo assim a utilização eficiente da informação. 


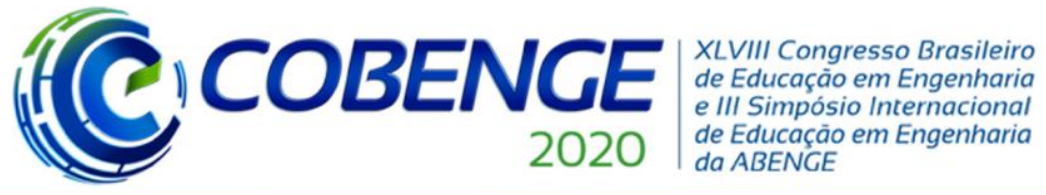

"Os desafios para formar hoje o engenheiro do amanhã"

Por isso a definição da plataforma de trabalho assim como a rede e pacotes de softwares se tornam um aspecto essencial quando se trabalha com essa metodologia. Pode-se citar alguns softwares voltados para a metodologia BIM existentes no mercado: O Vico Software da "Graphisoft@" que trabalha na mesma plataforma do "Archicad® ${ }^{\circledR}$ " e o "Revit ${ }^{\circledR} "$ da "Autodesk ${ }^{\circledR}$ " que trabalha na mesma plataforma do "AutoCAD®", sendo o último uns dos mais populares e utilizados devido sua associação com o "AutoCAD®" sendo ambos feitos pela mesma empresa.

Uma metodologia tão completa e em grande demanda possui uma grande quantidade de vantagens que para um bom contexto de sua importância devem ser citadas. Um grande benefício é a visualização tridimensional facilitadora da comunicação entre os diferentes intervenientes permitindo assim um melhor entendimento do projeto, além disso a pesquisa de conflitos entre os projetos de especialidade é muito facilitada pelos software BIM disponíveis no mercado, aumentando a qualidade dos projetos e diminuindo posteriores pedidos de informação em fase de obra. Outro préstimo do uso do BIM são as estimativas orçamentais mais rápidas e com menor margem de erro, que faz correlação com o arquivo de muita informação em torno de um modelo BIM que garante o acesso facilitado à informação, permanentemente atualizada e coordenada entre a equipe, reduzindo conflitos e erros de coordenação entre especialidades, aumentando assim a eficiência do projeto como um todo (VENÂNCIO, 2015).

O BIM proporciona grandes benefícios para a área da construção civil, sendo assim sua implementação de vital importância na formação acadêmica do curso de Engenharia Civil nos dias atuais. Muitas estratégias são discutidas para a efetivação da metodologia no curso, porém na maioria dos casos duas dessas estratégias tem maior destaque: Uma de forma integrada com as cadeiras do curso, dissolvendo assim o conteúdo e o método de ensino ao longo do tempo do curso, trazendo assim o uso do BIM para o dia a dia dos alunos e outra de forma mais pontual, com a criação de uma cadeira específica que deve ser cursada em algum momento durante a graduação, com cada uma possuindo suas vantagens e desvantagens (BARISON \& SANTOS, 2010, 2011).

Segundo Checcucci et al.(2013), a implementação integrada ao longo do curso de graduação em Arquitetura e Urbanismo se mostrou vantajosa utilizando o software Revit® como meio de ensino do BIM distribuídas em cadeiras mais focadas na área de projetos do curso, porém para isso tem-se em mente que é necessário uma grande quantidade de docentes e professores especializados na área, um desafio para a instituição. Além disso todo o processo de aplicação da metodologia é lento e pode levar uma quantidade de tempo considerável até atingir todo o potencial proposto.

Em contrapartida, a implementação pontual pode ser vantajosa em cursos de curta duração, similar a um tecnólogo. Devido ao pouco tempo de curso, uma abordagem mais incisiva e prática do ensino do BIM pode se tornar mais viável. Uma de suas desvantagens é a falta de integração com as cadeiras e disciplinas da graduação, diminuindo assim um pouco do possível aproveitamento da metodologia em correlação com as diversas áreas de estudo (CHECCUCCI, 2013).

A capacitação do docente é um quesito em comum nas duas estratégias, de acordo com Barison \& Santos (2010), é extremamente recomendado identificar quais são as responsabilidades, funções e competências dos especialistas em BIM que a instituição planeja disponibilizar para o curso e planejar a grade curricular de acordo com eles, para assim extrair 
(C) COBENGE

"Os desafios para formar hoje o engenheiro do amanhã"
$\mathrm{Ol}$ a $\mathrm{O} 3$ de dezembro Evento On-line

a maior eficiência do ensino da metodologia mesmo em cursos com uma implementação recente.

\section{METODOLOGIA}

A coleta dos dados foi feita por meio de questionários, com diversas perguntas relacionadas ao assunto as quais foram respondidas em uma escala de 1 a 5 , sendo 1 pouco relevante e 5 muito relevante. Tais questionários foram acessados por alunos (sem distinção de semestres) e professores (sem distinção de área) do curso via plataforma Google Forms. Cada grupo recebeu um questionário específico.

No questionário, inicialmente procurou-se saber informações sobre o participante conforme as Figuras 1 e 2.

Figura 1 - Primeira Questão Alunos.

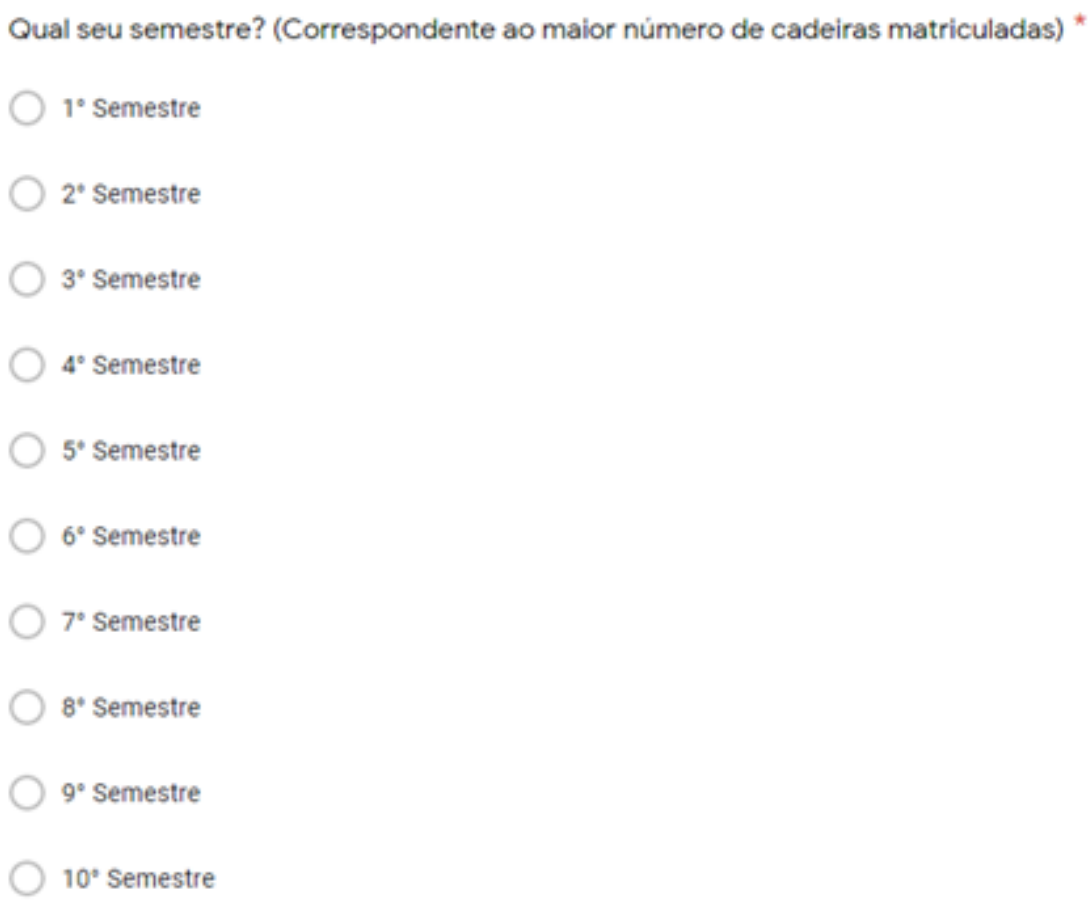

Fonte: Autoral (2020).

Figura 2 - Primeira Questão Docentes.

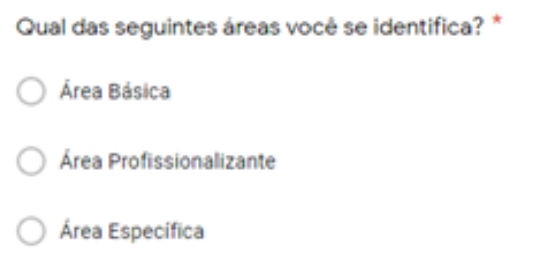

Fonte: Autoral (2020).

Em sequência, perguntou-se sobre a familiaridade de alunos e professores com a metodologia como visto na Figura 3. 


\section{COBENGE (C) COBENGE 2020 e III Simpósio Internacional de Educaçāo em Engenharia da ABENGE}

\section{"Os desafios para formar hoje o engenheiro do amanhã"}

Figura 3 - Segunda Questão Alunos/Docentes.

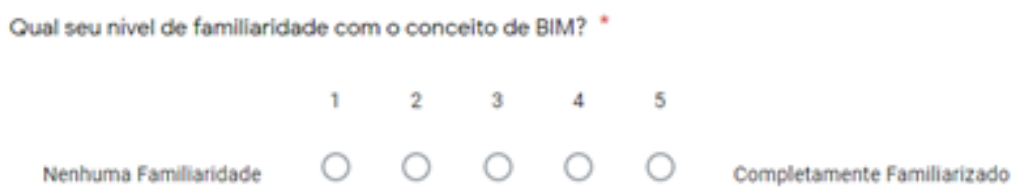

Fonte: Autoral (2020).

Em seguida, após breve explicação sobre BIM, tratou-se sobre a influência da metodologia na formação do discente e sua inserção no mercado de trabalho (Figura 4).

Figura 4 - Terceira Questão Alunos/Docentes.

\section{BIM (Building Information Modeling)}

BIM é um conceito que envolve a modelação das informações do edificio, criando um modelo digital integrado de todas as especialidades, e que abrange todo o ciclo de vida da edificaçăo. Com isso, pode-se determinar mais precisamente seu planejamento, levando em conta já no projeto os insumos da obra, os gastos, o cronograma de execuçăo e a integração de todos os projetos (executivo, hidráulico, etc), evitando incompatibilidades. Alguns dos softwares BIM utilizados hole em dia ş̃o Revit8, ARCHICADB e ALLPLANB, sendo estes pré-requisitos para determinadas vagas em grandes empresas e para submissăo de projetos em licitações públicas e regularizaçōes.

Dada esta breve explicação, qual a importância do aprendizado de BIM para a sua formação e inserção no mercado de trabalho?

$\begin{array}{lllllll} & 1 & 2 & 3 & 4 & 5 & \\ \text { Pouco Importante } & \bigcirc & \bigcirc & \bigcirc & \bigcirc & \bigcirc & \text { Muito Importante }\end{array}$

Fonte: Autoral (2020).

Aos alunos, foi perguntado se estes teriam interesse de tal abordagem em aula (Figura 5).

Figura 5 - Quarta Questão Alunos.

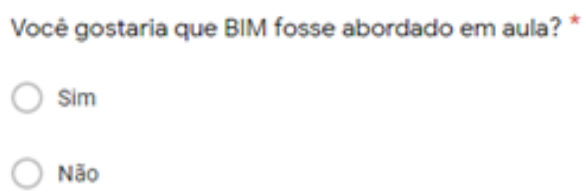

Fonte: Autoral (2020).

Após, foi abordado com os professores que ministram disciplinas para a graduação em Engenharia Civil se os mesmos possuem capacidade técnica para ministrar aulas sobre/com auxílio de BIM (Figura 6). Em caso de negativa deste, se estariam interessados e dispostos a se qualificarem em tal área, como visto na Figura 7. 


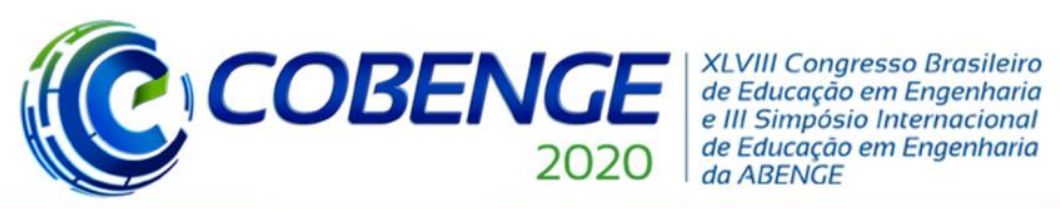

"Os desafios para formar hoje o engenheiro do amanhã"

Figura 6 - Quarta Questão Docentes.

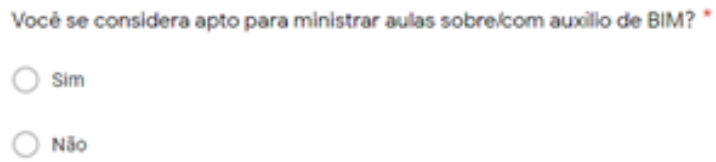

Fonte: Autoral (2020).

Figura 7 - Quinta Questão Docentes.

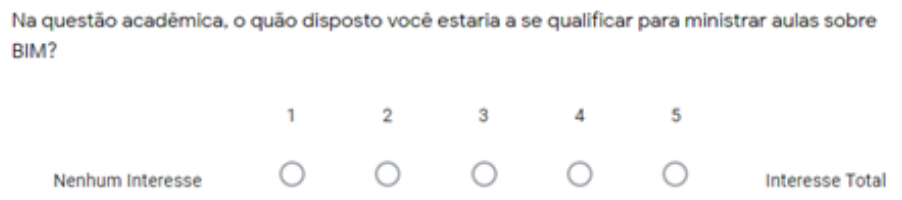

Fonte: Autoral (2020).

Por fim, foi questionado qual estratégia seria preferida: BIM em matérias isoladas ou ao longo do curso (Figura 8).

Figura 8 - Quinta Questão Alunos / Sexta Questão Docentes.

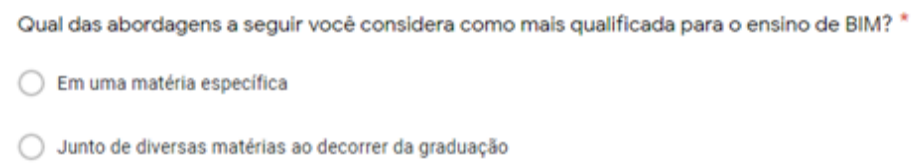

Fonte: Autoral (2020).

\section{RESULTADOS E DISCUSSÕES}

Participaram do questionário um total de 54 pessoas, sendo destes 45 alunos (aproximadamente $21,84 \%$ dos alunos do curso) e 9 docentes (aproximadamente $40,91 \%$ dos professores que ministram aulas para a Engenharia Civil).

Ao perguntar sobre o nível de familiaridade das categorias com o BIM, de acordo com a Tabela 1, as seguintes respostas foram obtidas:

Tabela 1 - Nível de Familiaridade.

\begin{tabular}{|c|c|c|c|c|}
\hline \multicolumn{5}{|c|}{ Nivel de Familiaridade } \\
\hline Nivel & Aluno & $\%$ Aluno & Docente & $\%$ Docente \\
\hline 1 & 9 & $20,0 \%$ & 4 & $44,4 \%$ \\
\hline 2 & 15 & $33,3 \%$ & 3 & $33,3 \%$ \\
\hline 3 & 13 & $28,9 \%$ & 1 & $11,1 \%$ \\
\hline 4 & 5 & $11,1 \%$ & 1 & $11,1 \%$ \\
\hline 5 & 3 & $6,7 \%$ & 0 & $0,0 \%$ \\
\hline
\end{tabular}

Fonte: Autoral (2019).

Ao comparar-se os dados, pode-se inferir que os alunos possuem maior familiaridade quando comparados com os docentes. Tal fenômeno pode ocorrer pelo maior contato dos alunos com o mercado de trabalho, haja vista que a utilização do BIM está sendo demandada pelo mesmo. 
(C) COBENGE

"Os desafios para formar hoje o engenheiro do amanhã"
$\mathrm{Ol}$ a $\mathrm{O3}$ de dezembro Evento On-line

Em seguida, pode-se observar na Tabela 2, tem-se o nível de importância do BIM para cada categoria:

Tabela 2 - Importância BIM.

\begin{tabular}{|c|c|c|c|c|}
\hline \multicolumn{5}{|c|}{ Nível de Importância } \\
\hline Nível & Aluno & $\%$ Aluno & Docente & $\%$ Docente \\
\hline 1 & 1 & $2,2 \%$ & 0 & $0,0 \%$ \\
\hline 2 & 0 & $0,0 \%$ & 0 & $0,0 \%$ \\
\hline 3 & 1 & $2,2 \%$ & 1 & $11,1 \%$ \\
\hline 4 & 6 & $13,3 \%$ & 3 & $33,3 \%$ \\
\hline 5 & 37 & $82,8 \%$ & 5 & $55,6 \%$ \\
\hline
\end{tabular}

Fonte: Autoral (2019).

Em sua maioria, ambas as categorias concordaram que o BIM é importante, demonstrando uma ressonância entre aquilo que o mercado está requisitando e a comunidade acadêmica demonstradas nas Figuras 9 e 10.

Figura 9 - Importância do BIM Alunos

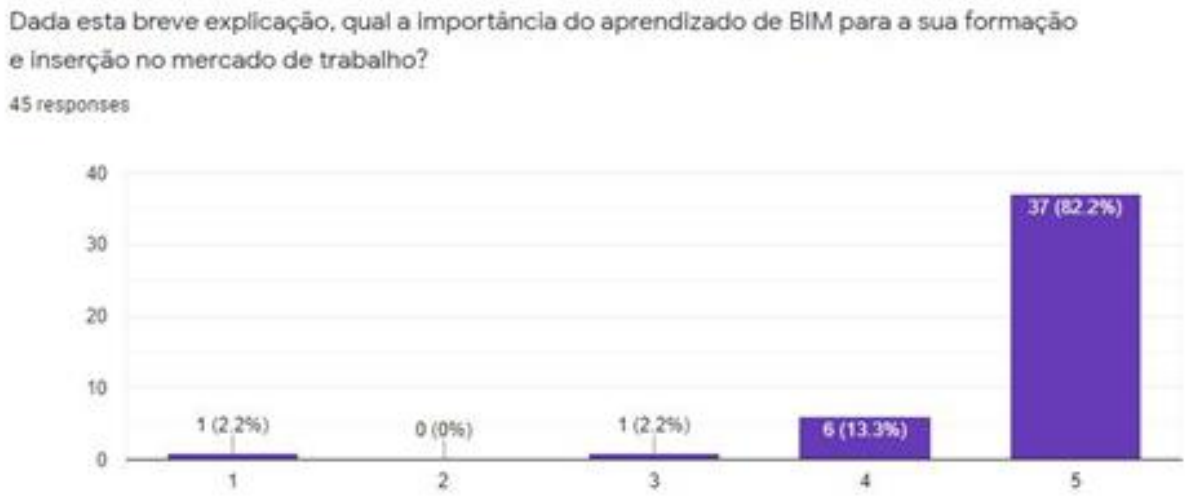

Fonte: Autoral (2019)

Figura 10 - Importância do BIM Docentes

Dada esta breve explicaçăo, qual a importáncia do aprendizado de BIM para a formaçăo e inserçào no mercado de trabalho do aluno?

9 responses

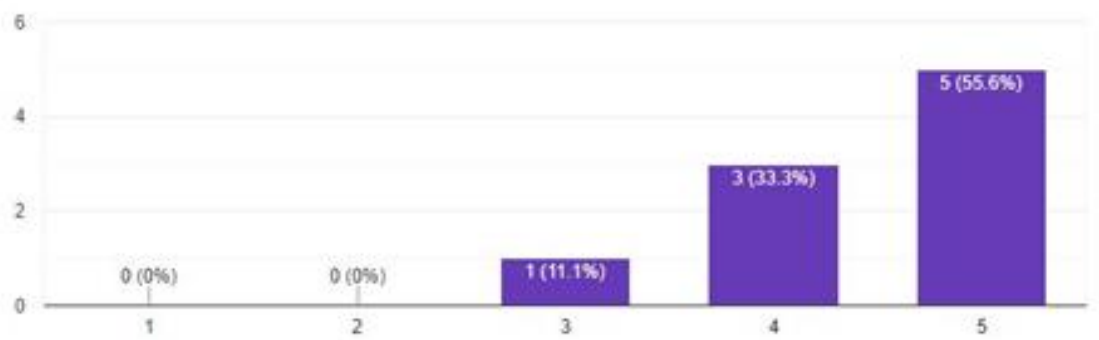

Fonte: Autoral (2019)

Tal constatação também é reforçada pela votação unânime no interesse da abordagem em aula pelos alunos demonstradas na Figura 11. 


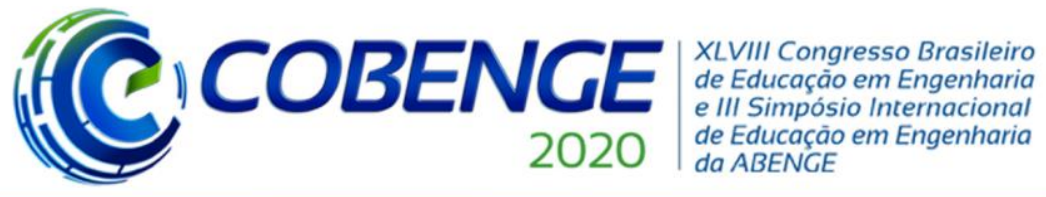

"Os desafios para formar hoje o engenheiro do amanhã"

Figura 11 - Interesse da Abordagem de BIM

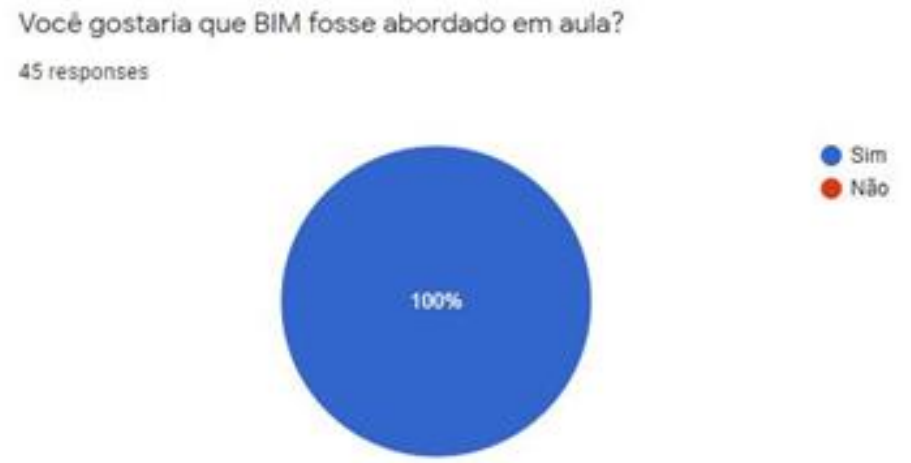

Fonte: Autoral (2019)

Como mostrado na Figura 12, a maioria dos docentes se mostram dispostos a se qualificar para ministrar aulas sobre BIM, haja vista que $88,9 \%$ destes haviam declarado não estar apto para tal atividade.

Figura 12 - Disposição para Qualificação dos Docentes.

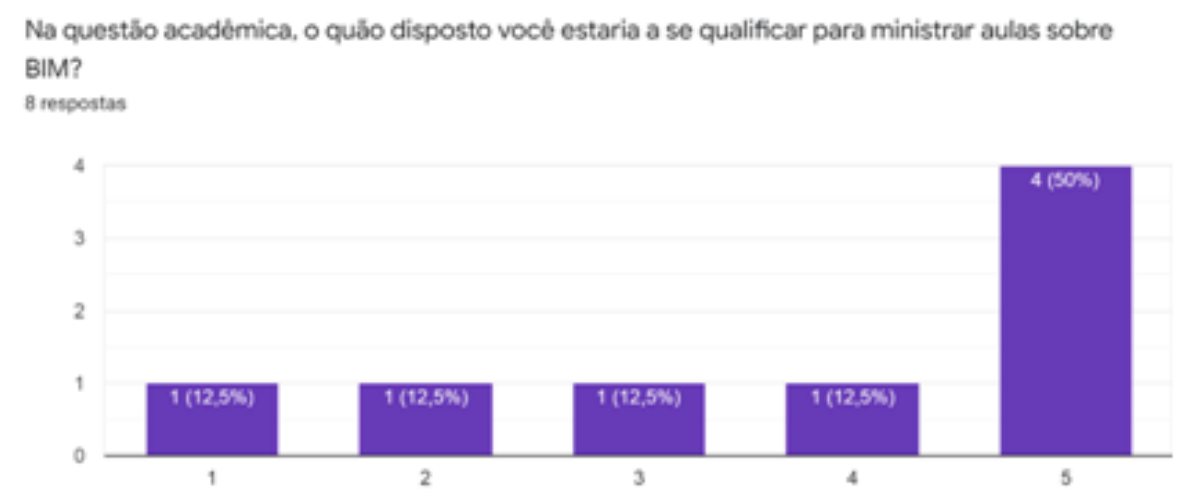

Fonte: Autoral (2019).

Referente a abordagem, 63\% dos consultados preferem aprender BIM junto de diversas matérias ao decorrer da graduação, em detrimento de em uma matéria específica, que obteve a votação correspondente de $37 \%$.

\section{CONCLUSÃO}

Pode-se concluir que o BIM já é encarado como assunto relevante entre a comunidade do curso, sendo capitaneado pela categoria discente desta. Tal liderança deve-se muito provavelmente ao contato dos alunos com o mercado de trabalho, que vem demandando o conhecimento desta metodologia pela sua poderosa ferramenta de planejamento e gestão na construção civil, principalmente por meio dos estágios requeridos ao decorrer da graduação. $\mathrm{O}$ apoio dos docentes neste quesito também é primordial, demonstrando que, apesar de estarem predominantemente no ambiente acadêmico, estão atentos às novidades da área, 
sendo de extrema importância para a atualização dos projetos pedagógicos de acordo com as necessidades identificadas.

Apesar do reconhecimento das partes envolvidas, se faz necessário a capacitação do corpo docente sobre BIM, sendo estes os responsáveis por transmitir os conhecimentos necessários para os estudantes ao decorrer de sua formação. $\mathrm{O}$ envolvimento da categoria docente será a chave para a implementação do BIM na graduação em Engenharia Civil, visto que o processo de aprendizagem de novas ferramentas e tecnologias podem ser encaradas como onerosas para alguns professores.

Com isso, percebe-se o surgimento de diversos pontos de estudo interessantes que podem ser melhor desenvolvidos em futuras pesquisas da área ou até mesmo uma continuação da discussão levantada ao longo do artigo. Algo interessante seria expandir a pesquisa para outras universidades, obtendo assim dados com mais precisão e um parecer mais apurado do estado da implementação metodologia em comparação com outras instituições.

Sabendo-se disso, espera-se encaminhar esta pesquisa como material de apoio e como manifestação da comunidade acadêmica do curso de Engenharia Civil ao seu colegiado, com o intuito de iniciar a discussão referente a implementação de uma ferramenta poderosa e vital como o BIM.

\section{REFERÊNCIAS BIBLIOGRÁFICAS}

AZEVEDO, J. O. (2009). Metodologia BIM - Building Information Modeling na Direcção Técnica de Obras. Tese de Mestrado, Universidade do Minho, Escola de Engenharia. Disponível em http://repositorium.sdum.uminho.pt/handle/1822/10695. Consultado em Outubro de 2019.

BARISON, M. B.; SANTOS, E. T. Review and analysis of current strategies for planning a BIM curriculum. Proc., CIB W78 2010 27th International Conference. Anais...2010 a Disponível em: < http://www.uel.br/pessoal/barison/Artigos_Tese/cibw78-2010.pdf>.Acesso em: 26 set. 2019.

BARISON, M. B.; SANTOS, E. T. Ensino de BIM: tendências atuais no cenário Internacional. Gestão \& Tecnologia de Projetos, São Carlos, v. 6, n. 2, p. 67-80, dez. 2011.

CHECCUCCI, É. DE S.; PEREIRA, A. P. C.; AMORIM, A. L. DE. Modelagem da Informação da Construção (BIM) no Ensino de Arquitetura. Proceedings of the XVII Conference of the Iberoamerican Society of Digital Graphics: Knowledge-based Design. Anais... In: SIGRADI 2013. Valparaiso, Chile: 2013

INBEC (Fortaleza - CE). Uso do BIM será obrigatório a partir de 2021 nos projetos e construções brasileiras. 2018. Disponível em: <https://www.inbec.com.br/blog/uso-bim-seraobrigatorio-partir-2021-projetos-construcoes-brasileiras>. Acesso em: 26 set. 2019.

VENÂNCIO, Maria João Lima. AVALIAÇÃO DA IMPLEMENTAÇÃO DE BIM BUILDING INFORMATION MODELING EM PORTUGAL. 2015. 402 f. Dissertação (Mestrado) - Curso de Engenharia Civil, Departamento de Engenharia Civil, Universidade do Porto, Porto, Portugal, 2015. 


\title{
Evento On-line
}

\section{IMPLEMENTATION OF BIM AT THE DEGREE IN CIVIL ENGIENEERING OF THE FEDERAL UNIVERSITY OF PELOTAS: A PEDAGOGIC APPROACH}

\begin{abstract}
The BIM methodology has been standing out for its practicality and gains, both in value and in planning time, in the development of civil construction projects. Being a competence required by big companies and the public power, the implementation of the teaching of such methodology during the undergraduate degree becomes more than needed for the formation of qualified and capable of acting with the most suited technological tools civil engineers, optimizing the intrinsic processes of the profession. With the proposal to insert BIM in the curriculum of the degree in civil engineering of the Federal University of Pelotas, the considerations about the methodology was investigated in the course's community. When comparing with the faculty, the students already had more familiarity with the concepts of BIM, probably due the more intense interaction with the labour market. All the course's academic community agreed that the teaching of BIM can promote the development of relevant competence for the students formation, in addition of the unanimous vote between the students for the option of having BIM presented in class. Most of the faculty were positive about qualifying themselves to teach classes about the methodology. The academic community preferred that BIM was taught throughout the degree, in various subjects. The need for faculty qualification and their support for a successful implementation of the methodology is essential, being these those capable of updating the course curriculum and engaging the rest of the community.
\end{abstract}

Keywords: BIM. Implementation. Curriculum. 